\title{
Feature Extraction from Mass Spectra for Classification of Pathological States
}

\author{
Alexandros Kalousis, Julien Prados, Elton Rexhepaj, and Melanie Hilario \\ University of Geneva, \\ Computer Science Department, \\ Rue General Dufour, 1211, Geneve, Switzerland \\ $\{$ kalousis, prados, hilario\}@cui.unige.ch, rexhepaj@unil.ch
}

\begin{abstract}
Mass spectrometry is becoming an important tool in proteomics. The representation of mass spectra is characterized by very high dimensionality and a high level of redundancy. Here we present a feature extraction method for mass spectra that directly models for domain knowledge, reduces the dimensionality and redundancy of the initial representation and controls for the level of granularity of feature extraction by seeking to optimize classification accuracy. A number of experiments are performed which show that the feature extraction preserves the initial discriminatory content of the learning examples.
\end{abstract}

\section{Proteomics and Mass Spectrometry}

Clinical proteomics aims at investigating changes in protein expression in order to discover new disease markers and drug targets. Mass spectrometry is emerging as an important tool for biomarker discovery in proteomics. To discover these biomarker patterns, the data miner must face a number of technical challenges, foremost among which is the extremely high-dimensionality and high redundancy of mass spectra.

In this paper we will present a method of feature extraction from mass spectra that retains as much as possible the initial information content of learning examples, extracts biologically meaningful features, reduces the degree of spatial redundancy, and achieves a significant level of dimensionality reduction.

One of the main problems in preprocessing and feature extraction from mass spectra is the appropriate selection of the preprocessing parameters. This is qualitative relying on systematic experimentation and visual inspection of the resulting spectra. There is a strong need for automatic and objective methods of parameter selection, [1. The problem is that it is not obvious what should be the measure that a given parameter set should optimize. However when the goal is classification and biomarker discovery one obvious measure to optimize is classification accuracy. We propose a solution that tightly couples the preprocessing and feature extraction steps with the learning task and uses the estimated classification performance of the latter to guide the selection of the appropriate set of parameters. In order to do that we exploit a common practice in machine learning in which cross-validation is used for parameter selection, 2. 
The paper is organized as follows: section 2 describes the preprocessing that we apply on a mass spectrometry problem; section 3 explains which steps of preprocessing, and how, are related with feature extraction and how to control the dimensionality in the new representation; in section 4 we investigate the degree of dimensionality reduction brought by feature extraction, we perform a series of classification experiments in order to establish its classification performance, and exhibit how we can automatically select the appropriate parameter values for preprocessing and feature extraction; finally we conclude in section 5 .

\section{Mass Spectra Preprocessing}

A mass spectrum of a biological sample is a one-dimensional signal. The $\mathrm{x}$-axis corresponds to the mass, $\mathrm{m} / \mathrm{z}$ value, of proteins detected in the biological sample and the $y$-axis to the intensity of these masses, the latter is strongly related to the concentration of the corresponding proteins in the sample. A mass spectrum is a vector whose dimensionality is equal to the number of $\mathrm{m} / \mathrm{z}$ values recorded by the spectrometer, the value of each dimension is the intensity of the corresponding $\mathrm{m} / \mathrm{z}$ value. Intensities of neighboring $\mathrm{m} / \mathrm{z}$ values are highly correlated, resulting in high spatial redundancy among the features of a mass spectrum.

Mass spectra demand considerable effort for preprocessing, which can be roughly divided to: baseline removal, denoising, smoothing, normalization, peak detection and calibration, [1. We will describe now how we tackled each of them.

The baseline is an offset of the intensities of masses, which happens mainly at low masses, and varies between different spectra. In order for comparisons between intensities of $\mathrm{m} / \mathrm{z}$ values to be meaningful it has to be subtracted. To compute the baseline we used a local weighted quadratic fitting on the list of the local minima extracted from the spectrum. On the new fitted values of local minima a new search for local minima was performed, the first fitting smooths out small variations. Using the new local minima the signal is split to piecewise constant parts and the final baseline is simply computed by the reapplication of the initial local weighted quadratic fitting, on the piecewise constant signal.

To denoise and smooth the signal we used wavelet decomposition coupled with a median filter; a detailed description is given in section 3. Signal intensities are normalized, to be less dependent on experimental conditions, via total ion current which is equivalent to normalizing with the $L_{1}$ norm of the spectrum.

Peak detection is the detection of local maxima in the mass spectrum. A peak collectively represents all the $\mathrm{m} / \mathrm{z}$ values that define it, that is: starting from its left closest local minimum and moving to its right closest local minimum. The intensities of all these neighboring $\mathrm{m} / \mathrm{z}$ values exhibit a high level of redundancy, thus by representing a spectrum only via its peaks we considerably reduce the level of spatial redundancy. Peak calibration establishes which peaks among different spectra correspond to the same peak, i.e. the same protein. We used the approach followed in 3] which is essentially complete linkage hierarchical clustering with some additional domain constraints. The final clusters contain masses from different spectra that correspond to the same peak. 


\section{$3 \quad$ Feature Extraction}

Feature extraction is the combined effect of all the preprocessing steps. However three of them are central: denoising-smoothing, peak detection and peak calibration. The first step determines how many peaks-features will be preserved in the preprocessed spectrum, it thus, indirectly, determines the dimensionality of the finally constructed feature space (later in this section we will see how this is achieved). The two latter steps are the actual steps of feature extraction. Clusters established in the peak calibration step become the extracted features. The feature value of a learning instance in the new representation for the feature that is associated with a given cluster will be simply the intensity in the preprocessed mass spectrum at the mass value that is associated with that cluster.

Wavelets are very popular in signal processing because they are able to analyze both local and global behavior of functions, a phenomenon dubbed time and frequency localization [4. Classical tools like the Fourier transform are of global nature and assume stationary signals, which is not the case for the signals found in mass spectra. This is why wavelets have recently received attention as a tool for preprocessing mass spectra [1. Wavelet decomposition reconstructs a signal as a linear combination of some basis functions. By thresholding the coefficients of the basis functions we get a denoised version of the signal.

We work with the decimated wavelet transform and perform the wavelet decomposition using Mallat's pyramid algorithm, 4]. As wavelet basis we have chosen Daubechies, which has been reported previously to have a good performance on mass spectrometry data, [1. We have opted for hard thresholding and in order to further smooth the produced signal we apply a moving median window filter (window size equals nine points). Smooth behavior is essential for peak detection, if the signal is not smooth enough a very high number of local maxima would be detected that are rather the result of random fluctuations.

To perform the thresholding we create the distribution-histogram of the wavelet coefficients. By specifying a percentile on that distribution, all the coefficients falling within it will be set to zero and the signal will be reconstructed from the remaining coefficients. The threshold of the wavelet coefficients controls the dimensionality of the finally produced feature space after peak detection. Large values of the threshold result in fewer detected peaks and thus lower dimensionality. The question is how to select the wavelet threshold without relying on a visual and qualitative inspection of the resulting signals. We should strive for a careful balance; as values of the wavelet threshold become higher and higher we do not remove only noise but we also start removing a part of the signal that potentially contains valuable discriminatory information.

\section{Experimentation}

We worked with three different mass spectrometry datasets, one for ovarian cancer [5], (version 8-07-02), one for prostate cancer [6] and an extended version of the early stroke diagnosis dataset used in [3]. A short description of these datasets is given in table 1 . They are all two class problems, diseased vs controls. 
The learning algorithms used are: a decision tree algorithm $\mathrm{J} 48$, with $\mathrm{M}=2$, $\mathrm{C}=0.25$, a one nearest neighbor algorithm, IBL, and a support vector machine algorithm, SMO, with a simple linear kernel and $\mathrm{C}=0.5$. The implementations of the algorithms were those of the WEKA machine learning environment, [7]. The three learning algorithms were chosen so that they represent a diverse set of learning biases. Performance estimation was done using 10-fold stratified crossvalidation and controlling for significant differences using McNemar's test with a level of significance of 0.05 .

We explored three issues: the degree of dimensionality reduction achieved by the feature extraction mechanism, the amount of discriminatory information preserved by the different levels of preprocessing, namely denoising and feature extraction, and finally how preprocessing could be optimized.

Dimensionality Reduction. We varied the wavelet threshold from 0.5 to 0.95 with a step of 0.05 , and from 0.95 to 0.99 with a step of 0.01 . The degree of dimensionality reduction ranges from $60 \%$ to $95 \%$ of the initial number of features in the complete mass spectrum depending on the threshold and the dataset. Due to lack of space we list some of the results in table 2, The dimensionality reduction is done in such a way that it reflects domain knowledge and reduces the spatial redundancy of the initial representation.

Table 1. Description of mass spectrometry datasets considered

\begin{tabular}{l|c|c|c|c}
\hline dataset & \# controls & \#diseased & mass range (Daltons) & \# features \\
\hline ovarian & 91 & 162 & $0-20 \mathrm{k}$ & 15154 \\
prostate & 253 & 69 & $0-20 \mathrm{k}$ & 15154 \\
stroke & 101 & 107 & $0-70 \mathrm{k}$ & 28664 \\
\hline
\end{tabular}

Table 2. Feature reduction for different values of the wavelet threshold. For each dataset and wavelet threshold, $\theta$, we give: the number of features after feature extraction (\# features), and the percentage of feature reduction (reduction \%).

\begin{tabular}{l|c|c|c|c|c|c}
\hline & \multicolumn{2}{|c|}{ prostate } & \multicolumn{2}{c|}{ ovarian } & \multicolumn{2}{c}{ stroke } \\
\hline$\theta$ & \# features & reduction \% & \#features & reduction \% & \#features & reduction \% \\
\hline 0.5 & 3779 & 75.06 & 1591 & 89.50 & 11983 & 58.19 \\
0.6 & 3538 & 76.65 & 1371 & 90.95 & 11294 & 60.59 \\
0.7 & 3223 & 78.73 & 991 & 93.46 & 9780 & 65.88 \\
0.8 & 2616 & 82.73 & 865 & 94.29 & 6954 & 75.73 \\
0.9 & 1668 & 88.99 & 775 & 94.88 & 3154 & 88.99 \\
0.99 & 1009 & 93.34 & 668 & 95.59 & 1255 & 95.62 \\
\hline
\end{tabular}

Preprocessing and Discrimination. To see whether the various steps of preprocessing preserve the discriminatory information we evaluated the learning algorithms on three different representations of the classification problems: 1) the 
initial complete mass spectra where we only performed baseline removal and normalization, bl-tic, this is a single dataset; 2) the complete mass spectra where baseline removal, normalization, noise removal with different wavelet thresholds, and smoothing are performed, this is a group of datasets collectively identified as all, each dataset corresponds to a specific wavelet threshold; 3) the datasets produced after feature extraction, collectively identified as peaks. bl-tic will provide us with a performance baseline since it contains all the initially available information. Comparing the performance of learning on all and bl-tic we can see how denoising and smoothing affects the discriminatory content of the learning examples, while comparisons between peaks and bl-tic allows us to establish the effect of feature extraction on the discriminatory content. The estimated performances (accuracies) are given in figure 1]

A close examination of figure 1 shows that in general the accuracies of the learning algorithms on the all and peaks representations are similar to the baseline accuracy on bl-tic. There is no clear trend associated with the level of denoising, and no systematic difference that would show a clear advantage or disadvantage of denoising-smoothing and feature extraction.

To establish a precise picture of the effect of denoising-smoothing and feature extraction on discrimination content we computed the significance level of the accuracy differences on the bl-tic representation and on each of the all, and peaks representations, i.e., for each value of the wavelet threshold, the results are summarized in table 3 in terms of significant wins and losses.

Table 3. Significant wins and losses table summarized over the different threshold values. A triplet $\mathrm{w} / \mathrm{t} / \mathrm{l}$ for a pair of representations $x$ vs $y$ gives the number of significant wins $(\mathrm{w})$, ties (t), and significant losses for $x$.

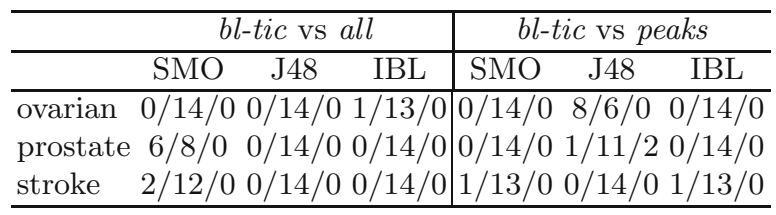

Denoising and smoothing in general preserve the discriminating information contained within the learning examples, table 3, column bl-tic vs all. However there are threshold values for which classification accuracy significantly deteriorates compared to the baseline; a fact that calls for an informed way of selecting the appropriate threshold value. A similar picture arises when we examine the performance of feature extraction for the different values of the wavelet threshold, table 3. column bl-tic vs peaks. In most of the cases feature extraction retains a discriminatory content similar to that of the bl-tic representation, nevertheless here also the wrong choice of the wavelet threshold can lead to a significant drop in classification accuracy compared with the baseline accuracy.

The optimal value of the wavelet threshold depends not only on the dataset but also on the learning algorithm used. A good or bad selection of the wavelet 

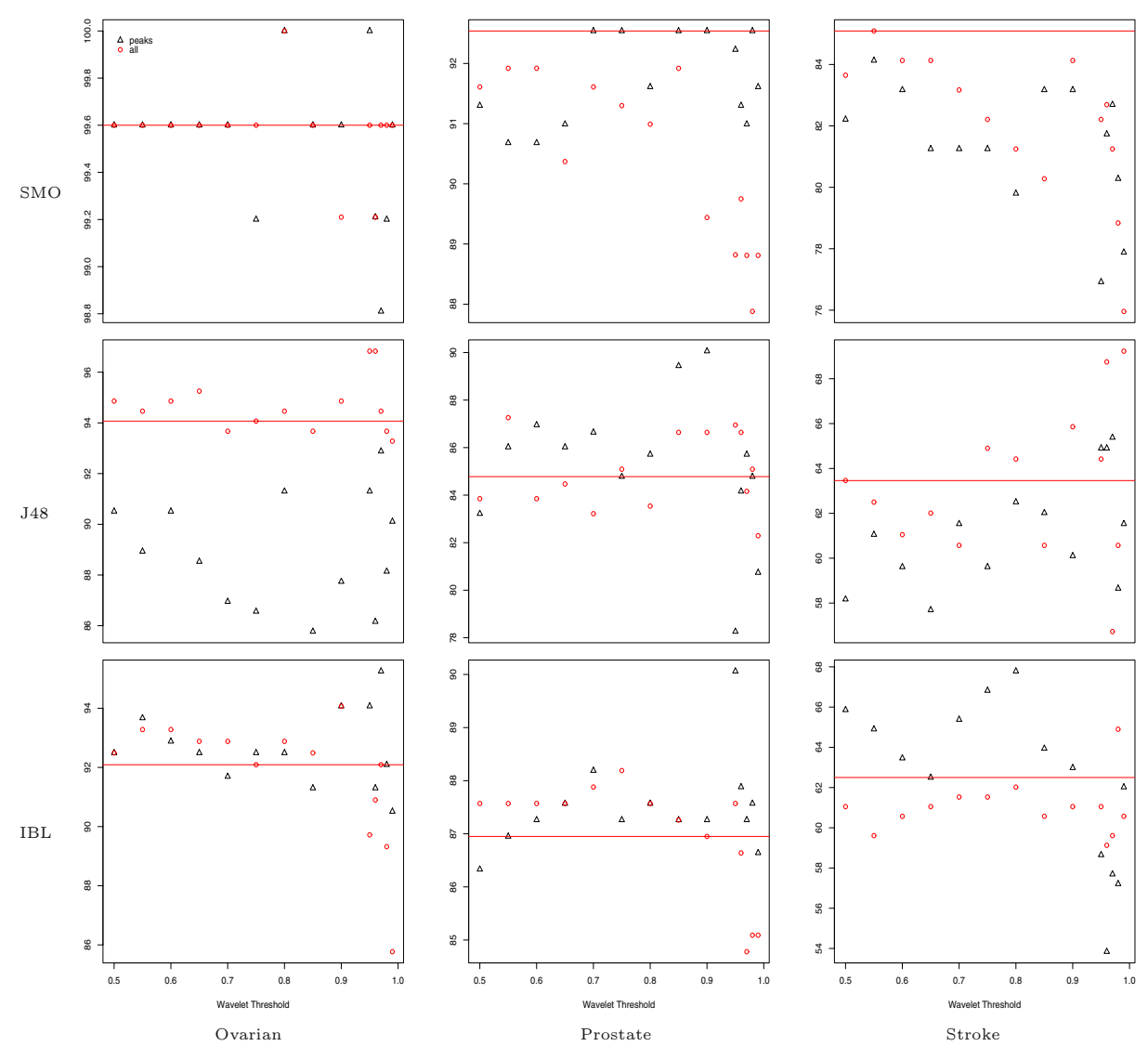

Fig. 1. Accuracy results for each dataset and learning algorithm. Each graph gives the accuracy of a given algorithm for all wavelet thresholds using: the complete spectrum, all, only the peaks, peaks. The horizontal lines correspond to the accuracy on the bl-tic version of the dataset. The y-axis gives the accuracy $\%$, note the differences in scales.

threshold can lead to a performance that is, respectively, significantly better or worse than the baseline performance. Thus all these raise again the issue of the optimal selection of the wavelet threshold in order to optimize feature extraction. Clearly this selection should be guided not only by the dataset but also by the learning algorithm that we are planning to use for classification.

Optimizing Denoising and Feature Extraction. We would like to select that wavelet threshold that would have the highest chances of maximizing classification performance on the testing instances for a given learning algorithm. To achieve that we tightly integrate the whole preprocessing pipeline to the learning process and adopt a commonly used technique for parameter tuning that is based on cross-validation. More precisely the user gives an initial list of interesting parameter values and selects a learning algorithm. Then as a part of 
the training phase the preprocessing and the learning algorithm are cross validated (ten folds) tightly coupled for each value. The value that gave the highest accuracy is chosen, the training set is preprocessed with the chosen value, the learning algorithm is trained on the preprocessed training set and tested on the test set.

Evaluation was done with ten fold stratified cross validation. The threshold values among which selection is performed are the same as in the previous experiments. For each dataset again two representations were considered: all that contains all the features where only denoising and smoothing is performed, and peaks where feature extraction was also performed, results are given in table 4.

Denoising with automatic selection of the appropriate wavelet threshold retains the discriminatory information of the learning examples when compared with the bl-tic representation. For any learner and any dataset, classification performances on the all representation are very similar with those on the $b l$ tic representation, no statistically significant differences are observed (statistical significance data not shown). The same holds when we compare the classification performance of feature extraction with the classification performance on bl-tic, performances are similar and no statistical significant difference is observed.

Table 4. Classification accuracy with automatic parameter selection. Automatic parameter selection is only performed for all and peaks, bl-tic is repeated for comparison reasons.

\begin{tabular}{l|lll|lcc|ccc}
\hline dataset & \multicolumn{3}{|c|}{ bl-tic } & \multicolumn{3}{c|}{ all } & \multicolumn{3}{c}{ peaks } \\
\hline & SMO & J48 & IBL & SMO & J48 & IBL & SMO & J48 & IBL \\
\hline Ovarian & 99.60 & 94.07 & 92.09 & 99.20 & 95.25 & 96.04 & 99.20 & 92.49 & 92.88 \\
prostate & 92.54 & 84.78 & 86.95 & 92.23 & 86.95 & 86.95 & 92.54 & 84.78 & 90.06 \\
stroke & 85.09 & 63.46 & 62.50 & 81.73 & 65.86 & 56.73 & 81.73 & 62.01 & 62.01 \\
\hline
\end{tabular}

Overall automatic selection of the wavelet threshold avoids the pitfalls of manual selection. It reduces the chances of selecting a threshold value that would result in a significant deterioration of the classification performance. Equally important it eliminates the need for a visual and qualitative inspection of the results of denoising in order to select the appropriate threshold, thus relieving the analyst from a significant burden, while on the same time it replaces a qualitative approach (visual inspection) with an objective criterion (classification accuracy).

\section{Conclusion}

Mass spectrometry data are characterized by very high dimensionality and very high levels of redundancy among their features. In this paper we exploited domain knowledge in order to reduce dimensionality and on the same time remove redundancy from the initial representation by feature extraction. One of our central tools was wavelet decomposition. Wavelets have been used before in 
mass spectrometry [8]9] and in other domains like measuring time series similarities [10]. Nevertheless they all worked with the wavelet coefficients. We return to the initial representation of the signal and extract features from that, thus the extracted features are of direct biological relevance. [1] use wavelets to extract peaks from mass spectra but they did not follow up with classification, moreover they stressed the problem of the appropriate level of denoising and parameter selection. To address these issues we tightly coupled feature extraction with classification and provided an effective and automatic way to control the granularity of feature extraction with a view to maximizing classification accuracy.

We should note here that what we propose is not a feature selection method. Our goal is not to minimize the number of features that can be used to effectively perform classification but to extract a high level, more compact, less redundant and well understood representation of the mass spectra that retains as much as possible the initial discriminatory content of the training examples. The new extracted representation seems, according to the experimental evidence, to retain the discriminatory content of the learning examples. Once the new representation is extracted one may proceed to a typical data analysis scenario where aggressive methods of feature selection could now be used on the new representation.

\section{References}

1. Morris, J., Coombes, K., Koomen, J., Baggerly, K., Kobayashi, R.: Feature extraction and quantification for mass spectrometry in biomedical applications using the mean spectrum. Bioinformatics (2005) Advanced publication.

2. Hastie, T., Tibshirani, R., Friedman, J.: The Elements of Statistical Learning. Springer (2001)

3. Prados, J., Kalousis, A., Sanchez, J.C., Allard, L., Carrette, O., Hilario, M.: Mining mass spectra for diagnosis and biomarker discovery of cerebral accidents. Proteomics 4 (2004) 2320-2332

4. Mallat, S.: A wavelet tour of signal processing. Academic Press (1999)

5. Petricoin, E., et al: Use of proteomic patterns in serum to identify ovarian cancer. The Lancet 395 (2002) 572-577

6. Petricoin, E., et al: Serum proteomic patterns for detection of prostate cancer. Journal of the NCI 94 (2002)

7. Witten, I., Frank, E.: Data Mining: Practical Machine Learning Tools and Techniques with Java Implementations. Morgan Kaufmann (1999)

8. Qu, Y., et al: Data reduction using a discrete wavelet transform in discriminant analysis of very high dimensional data. Biometrics 59 (2003) 143-151

9. Lee, K.R., Lin, X., Park, D., Eslava, S.: Megavariate data analysis of mass spectrometric proteomics data using latent variable projection method. Proteomics 3 (2003)

10. Zbigniew R. Struzik, A.S.: The haar wavelet transform in the time series similarity paradigm. In: Principles of Data Mining and Knowledge Discovery, Third European Conference, Springer (1999) 12-22 